\title{
Effects of Non-orthogonal Scheme on Error Modulation of Single-axis Rotating Strapdown INS
}

\author{
Wang Qichao $^{1, a}$, Wu Meiping ${ }^{1, b}$, Cai Shaokun ${ }^{1, c}$ and Chen Changfeng ${ }^{1, d}$ \\ ${ }^{1}$ College of Mechatronics and Automation, National University of Defense Technology, Changsha \\ 410073, China. \\ awangqichaonudt@163.com, ’bgfjwmp@163.com, ccsk527@163.com, ${ }^{\mathrm{d}} \mathrm{zg}$ jpjccf@163.com
}

Keywords: Non-orthogonal scheme, single-axis rotating, strapdown INS, error modulation.

\begin{abstract}
To improve the accuracy of strapdown inertial navigation system(SINS), a non-orthogonal installation scheme of inertial sensors was put forward for single-axis rotating SINS in this paper. Firstly, the principle of single-axis rotation modulation technology was expounded based on the error model of inertial sensors. Then, the effects of non-orthogonal scheme upon rotating modulation were analyzed theoretically, which show that the equivalent error magnitudes vary with angles between sensitive axes of inertial sensors. Finally, the simulation was performed. Experimental results show that the velocity error reduced from $1.91 \mathrm{~m} / \mathrm{s}$ to $1.03 \mathrm{~m} / \mathrm{s}$ in north and from $1.95 \mathrm{~m} / \mathrm{s}$ to $1.08 \mathrm{~m} / \mathrm{s}$ in east, and the positioning accuracy improved from $7.32 \mathrm{n}$ mile to $2.00 \mathrm{n}$ mile in north and from $5.06 \mathrm{n}$ mile to $1.70 \mathrm{n}$ mile in east within $24 \mathrm{~h}$ when suing the new scheme.
\end{abstract}

\section{Introduction}

As an important way of improving the accuracy of SINS, the rotation modulation technique is employed to modulate the errors of inertial sensors into periodically varied signals, and, as a result, it can remove the constant bias, slowly vary errors of the sensors and maintain a sufficient accuracy for long time [1]. Since the basic principle of rotation modulation technique for inertial navigation system based on ring laser gyro is studied by Levinson $\mathrm{E}$ in 1980, the error model of SINS, the effect of rotation modulation technique on accuracy improvement and different rotation schemes have been researched by many scholars at home and abroad. However, almost all the researches are based on the condition that the axes of sensors are orthogonal [2-4].

Considering this, in this paper, a non-orthogonal installation scheme is proposed in order to improve the accuracy of SINS. The principle of the rotation technique is introduced based on the measurement error models of sensors. Then, the effects of the new scheme on error modulation are discussed in detail, and numerical values of errors are compared. Finally, the simulation is performed and navigation accuracy is analyzed.

\section{Basic principle of rotation modulation technique}

Formulation of the principle of the rotation modulation technique involves the use of several coordinate frames, which are designated by the symbols "p", "s", "b”, and "n”. The p-frame is a nonorthogonal frame, whose axes are defined by the real directions of the sensitive axes of sensors. The s-frame, or rotating system, is a Cartesian system fixed to the rotating platform, and its z-axis is directed along the axis of rotation. The coordinate axes of b-frame are defined by the roll, yaw and pitch axes of the vehicle. The n-frame has its origin at the true position of the system, and its axes are directed toward true north, up and east.

The measurement values of the gyro cluster and the accelerometer cluster can be expressed as [5]

$$
\left\{\begin{array}{l}
W \delta_{i s}^{\delta}=\left(I+\delta \boldsymbol{K}_{g}\right)\left(\boldsymbol{I}+\delta \boldsymbol{C}_{g}\right) \boldsymbol{w}_{i s}^{s}+\boldsymbol{\varepsilon}+\boldsymbol{\varepsilon}_{n} \\
\mathcal{P}^{\circ}=\left(I+\delta \boldsymbol{K}_{a}\right)\left(\boldsymbol{I}+\delta \boldsymbol{C}_{a}\right) \boldsymbol{f}^{s}+\nabla+\nabla_{n}
\end{array}\right.
$$




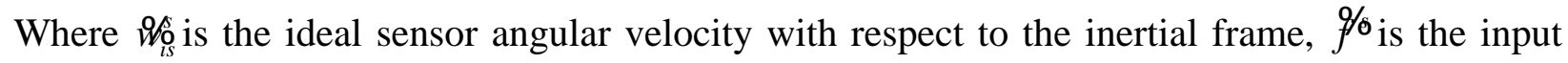
specific force expressed in s-frame, $\delta K_{g}$ and $\delta K_{a}$ denote the scale factor errors of the gyro and accelerometer, respectively, $\delta C_{g}$ and $\delta C_{a}$ are the misalignment of the gyro triad and accelerometer triad, $\varepsilon$ and $\nabla$ are the fixed biases of the gyro and accelerometer, $\varepsilon_{n}$ and $\nabla_{n}$ denote the noises of the gyro and accelerometer, respectively.

Ignoring the error product terms, Eq. (1) can be written as[6]

$\left\{\begin{array}{l}\delta \boldsymbol{w}_{i s}^{n}=\boldsymbol{C}_{s}^{n}\left[\left(\delta \boldsymbol{K}_{g}+\delta \boldsymbol{C}_{g}\right)\left(\boldsymbol{C}_{b}^{s} w_{i b}^{b}+\boldsymbol{w}_{b s}^{s}\right)+\boldsymbol{\varepsilon}+\boldsymbol{\varepsilon}_{n}\right] \\ \delta \boldsymbol{f}^{n}=\boldsymbol{C}_{s}^{n}\left[\left(\delta \boldsymbol{K}_{a}+\delta \boldsymbol{C}_{a}\right) \boldsymbol{f}^{s}+\nabla+\nabla_{n}\right]\end{array}\right.$

Assume that the IMU(inertial measurement unit,which constants of 3 gyros and 3 accelerometers) rotates around the yaw axis of the vehicle at rotation rate $w$. At $t$ epoch, the direction cosine matrix from s-frame to b-frame can be expressed as follows:

$C_{s}^{b}=\left[\begin{array}{ccc}\cos \omega t & 0 & \sin \omega t \\ 0 & 1 & 0 \\ -\sin \omega t & 0 & \cos \omega t\end{array}\right]$

Take the fixed bais for example and regardless of the other errors, the measurement values of gyro and accelerometer when rotating can be expressed as follows:

$$
\left\{\begin{array}{c}
\delta \boldsymbol{w}_{i s}^{n}=\left[\begin{array}{c}
\varepsilon_{x} \cos w_{c} t+\varepsilon_{z} \sin w_{c} t \\
\varepsilon_{y} \\
-\varepsilon_{x} \sin w_{c} t+\varepsilon_{z} \cos w_{c} t
\end{array}\right] \\
\delta \boldsymbol{f}^{n}=\left[\begin{array}{c}
\nabla_{x} \cos w_{c} t+\nabla_{z} \sin w_{c} t \\
\nabla_{y} \\
-\nabla_{x} \sin w_{c} t+\nabla_{z} \cos w_{c} t
\end{array}\right]
\end{array}\right.
$$

From the analysis above, we can note that the errors of sensors whose sensitive axes are perpendicular to rotating axis are modulated into periodic signals. And the conclusion comes that the navigation errors introduced by $\mathrm{x}$ and $\mathrm{z}$ axes are averaged out while the navigation errors introduced by y axis does not change.

\section{Effects of non-orthogonal scheme on rotating modulation}

The relation between p-frame and s-frame is depicted in Fig.1, where $\alpha_{1}, \alpha_{2}$ and $\alpha_{3}$ represent the angles between three axes of p-frame and their projection on $X_{b} O Z_{b}$ plane, respectively, $\beta_{1}, \beta_{2}$ and $\beta_{3}$ are angles between three projection axes and $X_{b}$ axis, respectively.

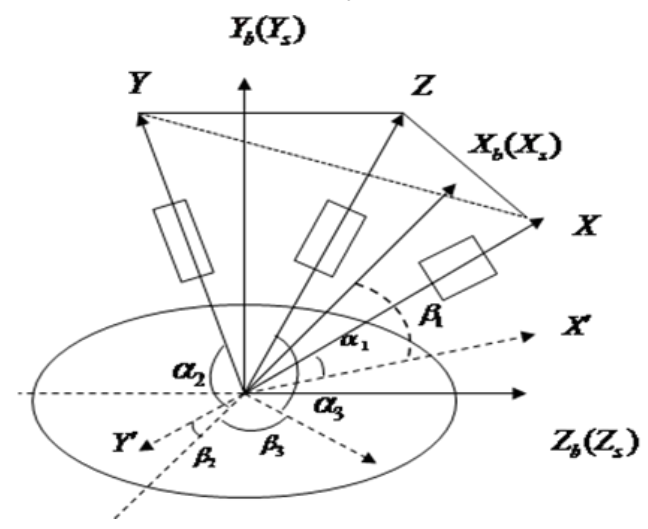

Fig. 1 Relations between coordinate systems

The projection matrix of p-frame in s-frame can be expressed as follows: 


$$
\left[\begin{array}{l}
f_{x}^{s} \\
f_{y}^{s} \\
f_{z}^{s}
\end{array}\right]=\left[\begin{array}{ccc}
\cos \alpha_{1} \cos \beta_{1} & -\cos \alpha_{2} \cos \beta_{2} & -\cos \alpha_{3} \cos \beta_{3} \\
\sin \alpha_{1} & \sin \alpha_{2} & \sin \alpha_{3} \\
\cos \alpha_{1} \sin \beta_{1} & -\cos \alpha_{2} \sin \beta_{2} & -\cos \alpha_{3} \sin \beta_{3}
\end{array}\right]\left[\begin{array}{l}
f_{x}{ }^{p} \\
f_{y} \\
f_{z}^{p}
\end{array}\right]
$$

The measurement value of pecific force in $n$-frame can be writen as

$$
\boldsymbol{f}^{n}=\boldsymbol{C}_{b}^{n} \boldsymbol{C}_{s}^{b} \boldsymbol{C}_{p}^{s} \boldsymbol{f}^{p}
$$

Assum that $C_{b}^{n}=I$ at the initial time and the following equations can be derived from Eq.(3), Eq.(5) and Eq. (6):

$$
\left[\begin{array}{l}
f_{x}^{n} \\
f_{y}^{n} \\
f_{z}^{n}
\end{array}\right]=\left[\begin{array}{ccc}
\cos \alpha_{1} \cos \beta_{1} \cos \omega t & -\cos \alpha_{2} \cos \beta_{2} \cos \omega t & -\cos \alpha_{3} \cos \beta_{3} \cos \omega t \\
+\cos \alpha_{1} \sin \beta_{1} \sin \omega t & -\cos \alpha_{2} \sin \beta_{2} \sin \omega t & -\cos \alpha_{3} \sin \beta_{3} \sin \omega t \\
\sin \alpha_{1} & \sin \alpha_{2} & \sin \alpha_{3} \\
\cos \alpha_{1} \sin \beta_{1} \cos \omega t & \cos \alpha_{2} \cos \beta_{2} \sin \omega t- & \cos \alpha_{3} \cos \beta_{3} \sin \omega t \\
-\cos \alpha_{1} \cos \beta_{1} \sin \omega t & \cos \alpha_{2} \sin \beta_{2} \cos \omega t & -\cos \alpha_{3} \sin \beta_{3} \cos \omega t
\end{array}\right]\left[\begin{array}{c}
f_{x}^{p} \\
f_{y}^{p} \\
f_{z}^{p}
\end{array}\right]
$$

Take the fixed bais of accelerometer for example, the measurement errors of pecific force can be expressed as follows:

$$
\left[\begin{array}{l}
\delta f_{x}^{n} \\
\delta f_{y}^{n} \\
\delta f_{z}^{n}
\end{array}\right]=\left[\begin{array}{c}
\left(\cos \alpha_{1} \cos \beta_{1} \cos w t+\cos \alpha_{1} \sin \beta_{1} \sin w t\right) \nabla_{x}-\left(\cos \alpha_{2} \cos \beta_{2} \cos w t+\right. \\
\left.\cos \alpha_{2} \sin \beta_{2} \sin w t\right) \nabla_{y}-\left(\cos \alpha_{3} \cos \beta_{3} \cos w t+\cos \alpha_{3} \sin \beta_{3} \sin w t\right) \nabla_{z} \\
\sin \alpha_{1} \nabla_{x}+\sin \alpha_{2} \nabla_{y}+\sin \beta_{3} \nabla_{z} \\
\left(\cos \alpha_{1} \sin \beta_{1} \cos w t-\cos \alpha_{1} \cos \beta_{1} \sin w t\right) \nabla_{x}+\left(\cos \alpha_{2} \cos \beta_{2} \sin w t-\right. \\
\left.\cos \alpha_{2} \sin \beta_{2} \cos w t\right) \nabla_{y}+\left(\cos \alpha_{3} \cos \beta_{3} \sin w t-\cos \alpha_{3} \sin \beta_{3} \cos w t\right) \nabla_{z}
\end{array}\right]
$$

According to Eq. (8), it can be found that the errors are still modulated into period signals with the same magnitude in north and east when using non-orthogonal installation. The pecific force errors are constrainted by six projection angels in north and east and three projection angels in up. Therefore, the projection angels can be obtained to make the equivalent error magnitudes as small as possible.

Using orthogonal experiment method for reference [7], five levels are selected for each projection angle. The results of equivalent error magnitudes are as follows:

Table 1 Comparison of error magnitudes caused by different projection angels

\begin{tabular}{cccccccccc}
\hline Numble & $\alpha_{1} /\left(^{\circ}\right)$ & $\alpha_{2} /\left(^{\circ}\right)$ & $\alpha_{3} /\left(^{\circ}\right)$ & $\beta_{1} /\left(^{\circ}\right)$ & $\beta_{2} /\left(^{\circ}\right)$ & $\beta_{3} /\left(^{\circ}\right)$ & $\mathrm{N}(\mathrm{mGal})$ & $\mathrm{E}(\mathrm{mGal})$ & $\mathrm{U}(\mathrm{mGal})$ \\
\hline 1 & 15 & 30 & 45 & 60 & 75 & 60 & 0.9497 & 0.9497 & 2.9319 \\
2 & 60 & 15 & 30 & 45 & 60 & 75 & 2.3660 & 2.3660 & 3.2497 \\
3 & 75 & 60 & 15 & 30 & 45 & 60 & 2.3660 & 2.3660 & 4.1815 \\
4 & 60 & 75 & 60 & 15 & 30 & 45 & 0.4122 & 0.4122 & 5.3960 \\
5 & 45 & 60 & 75 & 60 & 15 & 30 & 0.3660 & 0.3660 & 5.0781 \\
6 & 30 & 45 & 60 & 75 & 60 & 15 & 1.1840 & 1.1840 & 4.1463 \\
7 & 0 & 90 & 0 & 0 & 0 & 90 & 2.8284 & 2.8284 & 2.0000 \\
\hline
\end{tabular}

Table 1 shows that the equivalent error magnitudes of non-orthogonal frame are smaller than that of orthogonal frame in north and east, and the minimum is one-third of the maximum. It is also indicated that the equivalent error magnitudes of non-orthogonal frame are bigger than that of orthogonal frame in up, and the maximum is double of the minimum. Considering that the error of accelerometer in up has less effect on navigation accuracy, and the drift of gyro can be esimated with the accuracy better than $0.001\left({ }^{\circ}\right) / \mathrm{h}$, the non-orthogonal frame is feasible.

\section{Simulation and analysis}

The simulation is based on the error equations of SINS using RMT, which can be expressed as follows: 


$$
\left\{\begin{array}{l}
\delta \boldsymbol{v}_{e}^{\ell}=\left[\boldsymbol{f}^{n} \times\right] \boldsymbol{\psi}+\boldsymbol{C}_{b}^{n} \boldsymbol{C}_{s}^{b} \delta \boldsymbol{f}^{s}-\boldsymbol{C}_{b}^{n}[\boldsymbol{\phi} \times] \boldsymbol{C}_{s}^{b} \boldsymbol{f}^{s}-\left(2 \boldsymbol{w}_{i e}^{n}+\boldsymbol{w}_{e n}^{n}\right) \times \delta \boldsymbol{v}_{e}^{n}+\left(2 \delta \boldsymbol{w}_{i e}^{n}+\delta \boldsymbol{w}_{e n}^{n}\right) \times \boldsymbol{v}_{e}^{n}-\delta \boldsymbol{g}^{n} \\
\delta \boldsymbol{\beta}=\delta \boldsymbol{v}_{e}^{n} \\
\boldsymbol{\psi} \boldsymbol{\&}=\delta \boldsymbol{\omega}_{i n}^{n}-\boldsymbol{\omega}_{i n}^{n} \times \boldsymbol{\psi}-\boldsymbol{C}_{b}^{n} \boldsymbol{C}_{s}^{b} \delta \boldsymbol{\omega}_{i s}^{s}+\boldsymbol{C}_{b}^{n}[\boldsymbol{\phi} \times] \boldsymbol{C}_{s}^{b} \boldsymbol{\omega}_{i s}^{s}
\end{array}\right.
$$

Where $\delta \boldsymbol{v}_{e}^{n}$ is velocity error, $\delta \boldsymbol{p}$ is position error, and $\boldsymbol{\psi}$ is attitude error.

To verify the accuracy of different frames, parameters of simulation are set firstly. The constant drift rates of three gyros are set as $0.01\left(^{\circ}\right) / \mathrm{h}$. The constant biases of three accelerometers are set as $2 \mathrm{mGal}$. The stochastic noise in gyros and accelerometers are both Gaussian white noise, and respectively, the levels are set as $0.001\left({ }^{\circ}\right) / \mathrm{h}$ and $1 \mathrm{mGal}$. The scale factor errors of gyros and accelerometers are set as $1 \mathrm{ppm}$ and $5 \mathrm{ppm}$, respectively. The angle measurement error of platform is $1^{\prime}$.The initial angles of yaw, roll and pitch are $5^{\circ}, 5^{\circ}$ and $0^{\circ}$, respectively. The initial position is at latitude of $30^{\circ}$ and longitude of $110^{\circ}$. The platform rotates continuously at the rate of $6\left(^{\circ}\right) / \mathrm{s}[8]$.

The angle values of non-orthogonal frames are set as values of Row1, Row3 and Row5 in Table 1, respectively. The velocity error and positioning accuracy of different installation frames are compared through 24h simulation. The results of orthogonal and non-orthogonal schemes are compared in Fig. 2, and all the results are shown in Table. 2.
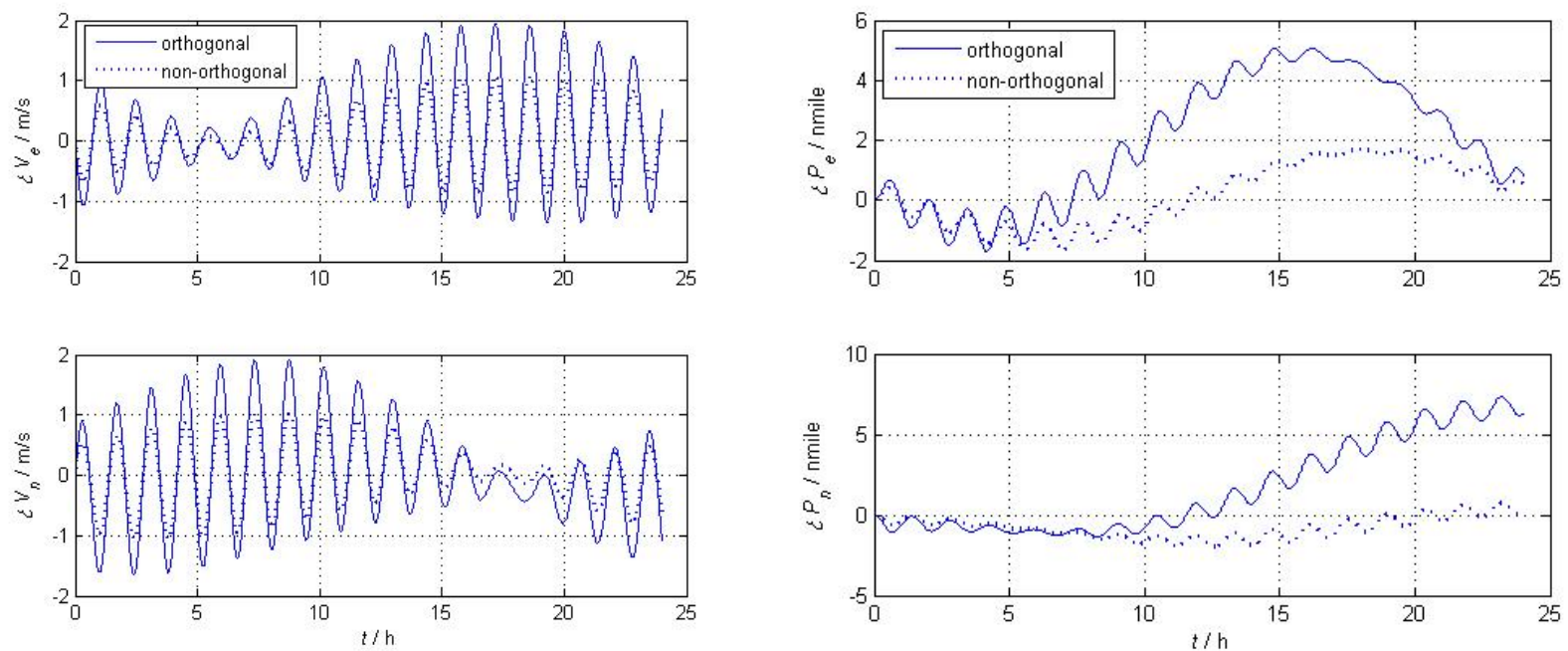

(a) Comparison of velocity errors

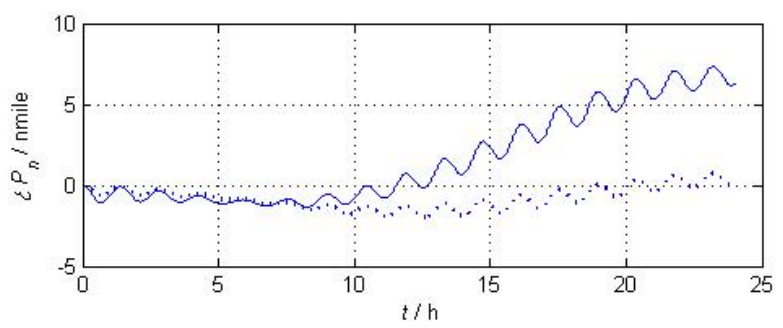

(b) Comparison of positioning errors

Fig. 2 Navigation errors of two different schemes Table 2 Velocity error and positioning accuracy of different schemes

\begin{tabular}{ccccc}
\hline Installation scheme & $\delta v_{e}(\mathrm{~m} / \mathrm{s})$ & $\delta v_{n}(\mathrm{~m} / \mathrm{s})$ & $\delta p_{e}(\mathrm{n}$ mile $)$ & $\delta p_{n}$ (n mile) \\
\hline Non-orthogonal scheme 1 & 1.48 & 1.39 & 2.35 & 2.90 \\
Non-orthogonal scheme 2 & 1.82 & 1.79 & 2.88 & 3.51 \\
Non-orthogonal scheme 3 & 1.08 & 1.03 & 1.70 & 2.01 \\
Orthogonal scheme & 1.95 & 1.91 & 5.06 & 7.32 \\
\hline
\end{tabular}

Fig. 2 and Table 2 show that both velocity error and positioning error reduced when using the non-orthogonal installation scheme. The velocity error reduced from $1.91 \mathrm{~m} / \mathrm{s}$ to $1.03 \mathrm{~m} / \mathrm{s}$ in north and from $1.95 \mathrm{~m} / \mathrm{s}$ to $1.08 \mathrm{~m} / \mathrm{s}$ in east, the positioning accuracy improved from $7.32 \mathrm{n}$ mile to $2.00 \mathrm{n}$ mile in north and from $5.06 \mathrm{n}$ mile to $1.70 \mathrm{n}$ mile in east.

\section{Summary}

The problem of non-orthogonal scheme of sensitive axes for single-axis rotating SINS is mainly studied in this paper. How the errors of inertial device are modulated is analyzed, and what is the effect of equivalent error magnitudes change on navigation accuracy is simulated. The simulation results show that the accuracy of navigation can be improved when suing the non-orthogonal scheme. It is also indicated that proper section of the angles between axes is very important. 


\section{References}

[1] Zhang L., Lian J., Wu M. Research on auto compensation technique of strapdown inertial navigation systems [C]. IEEE International Asia Conference on Informatics in Control, Automation and Robotics, Phuket, Thailand, 2009: 350-353.

[2] LEVINSON E, HORST J. The next generation marine inertial navigator is here now [C]// IEEE Position Location and Navigation Symposium. Las Vegas, NV, USA, 1994: 121-127.

[3] ISHIBASHI S, TSUKIOKA S, YOSHIDA H. Accuracy improvement of an inertial navigation system brought about by the rotational motion [C]// OCEANS 2007-Europe. Aberdeen, Scotland, United Kingdom, 2007: 1-5.

[4] Yang Y., Miao L. Fiber-optic strapdown inertial system with sensing cluster continuous rotation [J]. IEEE Transactions on Aerospace and Electronic Systems, 2004, 40(4): 1173-1178.

[5] Zhang H., Wu Y., Wu W. Improved multi-position calibration for inertial measurement units [J]. Measurement Science and Technology, 2010, 21(1): 1-11.

[6] Sun F., Sun W. Research on auto-compensation by rotation in strapdown inertial navigation systems [J]. Systems Engineering and Electronics, 2010, 32(1): 122-125. (in Chinese).

[7] Cai S. The research about airborne vector gravimeter and methods of errors separation [D]. Changsha: National University of Defense Technology, 2014.

[8] Zhang L., Lian J., Wu M., Hu X. An improved computation scheme of strapdown inertial navigation system using rotation technique [J]. Journal of central south university technology, 2012, 19(5): 1258-1266. 